\title{
Remote Operation and Assistance in Human Robot Interactions with Vibrotactile Feedback
}

\author{
Gabor Sziebig \\ Department of Industrial Engineering \\ UiT - The Arctic University of Norway \\ Narvik, Norway \\ gabor.sziebig@uit.no
}

\author{
Peter Korondi \\ Department of Mechatronics, Optics and Mechanical \\ Engineering Informatics \\ Budapest University of Technology an Economics \\ Budapest, Hungary \\ korondi@mogi.bme.hu
}

\begin{abstract}
This paper investigates intuitiveness in human robot interactions with focus on remote operation and assistance. The increasing number of robot applications in small volume production requires new techniques to ease the use of these sophisticated systems. A vibrotactile device was used for experiments. Vibration patterns are developed to represent a cognitive information channel for the remote operator. Most of the patterns are based on natural sensations, e.g. the feedback of gripping an object is slight vibration on the index finger and the thumb. Usability test results are presented. Intuitiveness of the vibrotactile feedback is confirmed in remote operation but the solution can be used in shop floor situations as well.
\end{abstract}

Keywords - industrial robotics; human robot interaction; remote operation; tactile feedback; usability test

\section{INTRODUCTION}

Communication between a human and a robot may take several forms, but these forms are largely influenced by whether the human and the robot are in proximity or not. Thus, Human Robot Interaction (HRI) can be separated into two general categories: proximity interactions and remote interactions. During proximity interactions the robots and the operators are co-located, while remotely accessing a robot cell means that the human and the robots are separated spatially and/or temporally [1, 2]. Remotely operated industrial robot systems provide faster and cheaper support irrespective of the geographical distances between the enterprise and the system integrator. Since more and more small and medium sized enterprises (SMEs) are integrating more complex robot systems [3] the need for knowledge transfer is increasing. The lack of proficiency in small companies raises a barrier for usability which should be solved in the near future. As the field of application for industrial robotics is expanding, both integrators and operators are facing the problem of efficient support; one possible answer is remote operation. Since remote interactions are limited new techniques should be used to transfer more important information from the robot cell to the remote user [4].

The paper consist of Section 2 describing the remote control in details and Section 3 presenting a vibrotactile feedback for remote control. The vibrotactile feedback was tested with users, the usability test and the results are presented in Subsection 4

\section{REMOTE CONTROL}

During remote control, one is physically separated from the system, hence the latter data, called Naturally Acquired Data (NAD) must be acquired and presented by an additional system, or it will be missed. Furthermore, NAD cannot be easily expressed by means of digits, letters, or other abstractive symbols, due to its symbolical nature [5].

Operating industrial robot systems locally, takes advantage of the human senses like hearing, seeing, smelling, force sensing/touch. The operator on site can also benefit from the ability of sense integration, small details can request attention which cannot be recreated on remote sites.

During remote operation when the operator is expected to operate out of office, this multi-modal communication is challenging due to the fact that there is lack of information provided to the remote operator. Traditional solutions have tried to use multiple camera systems, but the result has not been satisfactory due to the fact that multiple cameras make a very complex presentation on the remote monitor [5]. The challenges associated with this approach could be to determine which camera view is the one being used by the operator, whether the camera perspective in question causes confusion for the operator while the camera scenes should be well defined and the light conditions should be controlled. How to best solve the issues of the confusing control and camera perspectives in remote operation systems utilizing multicamera setups is considered an important topic for further work. If an operator confuses his/her perspective in a highly time-delayed situation and executes a planned motion, the use of a position-based control device could prove to be a potential hazard rather than a benefit in time-delayed operation.

Remote and local PCs are usually utilized for communication between remote and local room. Owing to existing transfer limitations and unpredictable delays of the present day Internet, special solutions must to be applied to protect the system. The most promising conception is to filter and compress the data before transferring, hence, even during very low transfer speed, crucial data can reach its destination. In case of sudden complete lack of Internet connection, the system should safely stop working. Information that needs to be transferred from industrial robot system to the remote operation system can be categorized into three groups from a functional standpoint [6]: 


\section{Safety}

The category of safety is intended to hold all information regarding the safety of both humans and equipment during remote operation. Information such as emergency stop signals, collisions, personnel entering the cell etc., are core information that should always be a part of this category. Depending on the task and its specifications, the category can also include taskspecific safety information. An example of this could be abnormally or dangerously high contact-forces during a grinding operation.

\section{Control}

The control category of the transfer channel is responsible for all information needed for efficient control of the remotely operated industrial robot system. Information such as robot control commands and monitoring for evaluating the state of the robot and its environment, are considered a part of the control category. Task and process specific control and monitoring information is also to be incorporated. Examples for a grinding process could be controls for the grinding motor, the contact-forces, surface temperature etc.

\section{Service, Setup \& Maintenance}

Service, setup \& maintenance, is the lowest prioritized category. Much of the information in this category does not have stringent requirements for delivery speed, and in some cases could be delivered on demand. Examples of information in this category could be the robot controller's constant settings, its robot programs, or diagnostic data from the robot or other equipment in the setup.

Humans are highly dependent on using all of their trained senses while performing everyday tasks. In robot operation, stereo and peripheral vision is important for depth perception and spatial awareness, which make the operator able to skilfully control the robot around obstacles and singularities. While in remote operation, these trained senses are limited or absent. In order to make his/her work feasible, the operator must be provided with certain data that in the local room would be naturally acquired by human senses. Without the additionally acquired data, one cannot imagine performing even simple robot tasks as guiding the robot to pick and place.

\section{VIBROTACTILE FEEDBACK}

There are many methods to get feedback from robots; this section aims to investigate a tactile feedback solution and gives an introduction to an in-house developed low-cost vibrotactile glove system.

A tactile device is a man-machine interface that can reproduce the tactile parameters such as the force, texture, temperature and shape, as truly as possible. The existent tactile devices are classified as pneumatic tactile, vibrotactile, electrotactile, etc. [7]

Generally, it is reported that human touch senses are poor at determining absolute quantities but very sensitive to changes [8], so tactile sensing, as an alternative method of achieving feedback has been considered in this paper.
Vibrotactile feedback is one of the user-friendliest features for operators [9]. For example users are using touch based cell phones everyday that usually use vibrotactile feedback for its ringtone, dial keypad to indicate whether the key is pressed or not.

Since humans use their hands for interactions and to manipulate objects, it is an appropriate body part to stimulate human touch sensors effectively. Additionally a vibrotactile glove is a low-cost and small-sized device that leads to having an interaction with fewer training for robot operators.

\section{A. Sensation in human skin}

Vibrotactile feedback describes sensations, which are applied to the skin and perceived by the human sense of touch including vibration, pressure, stretching, and touch. The tactile sensation process is a bidirectional exchange of information, making tactile sensation different from other sensation processes, such as visual sensation. Therefore, a tactile interface must allow users to interact actively in remote operation.

There are three types of receptors in the skin responsible for transferring mechanical solicitation (Figure 1):

- Merkel's receptors: high spatial resolution, slow adaption rate,

- Pacini's corpuscles: rapid vibration detection, fast adaption rate,

- Meissner's corpuscles: high spatial resolution, fast adaption rate, located in the glabrous skin (fingers, lips) [10].

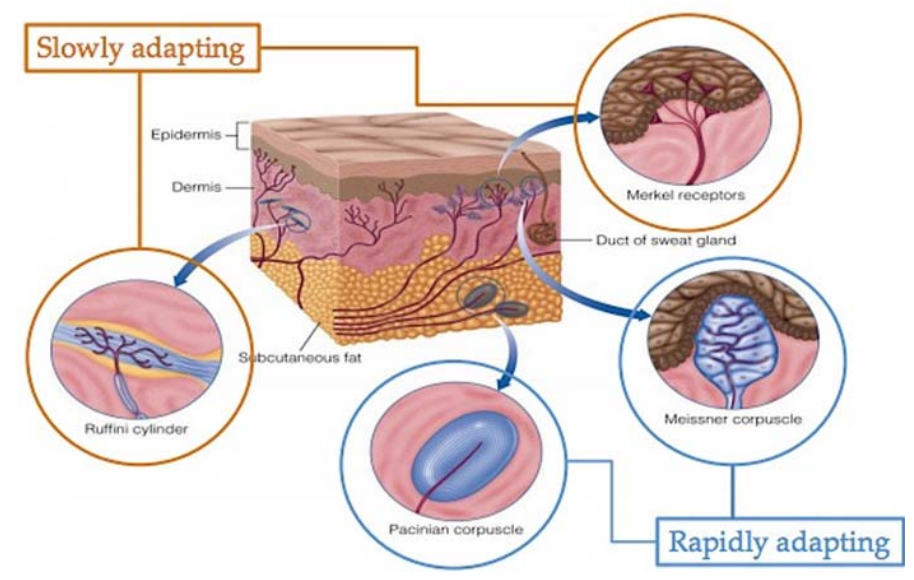

Fig. 1. Tactile receptors of human skin [11]

The information gathered by the receptors is transferred to the brain via the fastest communication channel in the human body: the dorsal lateral column way, where the information travels at the speed of $100 \mathrm{~m} / \mathrm{s}$. The sensing resolution of the skin is variable on the human body. Most precise points are on 
the fingers where the spatial resolution is of the order of millimetres. It is well known that the human skin hosts different type of mechanoreceptors. These mechanoreceptors are divided into two groups: slow-adapting and fast-adapting. The slow-adapting detects static force and the fast-adapting detects vibration and acceleration. The density of these receptors defines the spatial resolution of the skin in given positions (fingertips carry the most, palm the least) [10].

To make an effective vibrotactile stimulator, some main parameters such as the displacement and frequency of vibration, and the spacing of vibrated pins have to be considered.

\section{B. Vibrotactile glove system}

It is composed of a standard working glove with five vibration motors on different locations. Figure 2 depicts the locations of vibration motors on the hand and the composed system used in experiments. The total weight of the vibrotactile glove is 190 grams, the size of the Control Board is $70 \times 60 \times$ $40 \mathrm{~mm}$.

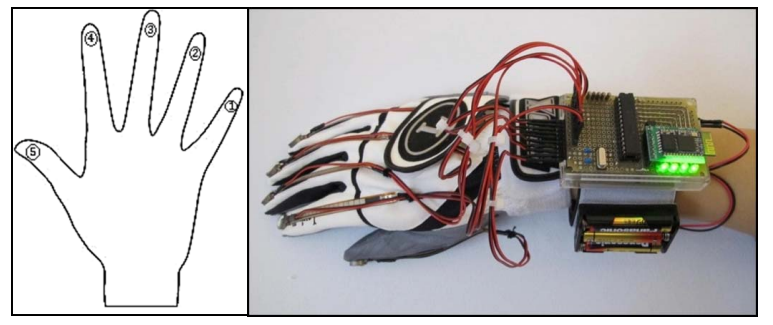

Fig. 2. Experimental vibrotactile glove system

Usage of vibration motors is only one of the available choices for small-sized tactile displays, which is suitable for an easy-to-use, highly portable and with lightweight interface design [12].

The intensity of the vibration, the vibration activation time and vibration pattern are important variables in feedback utilization. The intensity of the vibration stimulus can be set in different levels by changing the duty ratio of the Pulse Width Modulation (PWM).

\section{Cognitive vibrotactile feedback}

A set of robot cell events and parameters were chosen to be communicated to the user. During remote operation the operator has only a limited awareness of the environment due to the lack of communication channels. To overcome this bottleneck vibrotactile feedback may be used; the transferred information is encoded into vibration patterns. Using cognitive approach this encoding can be intuitive as the experiments proved. Time parameters of the vibration patterns' are summarized in Table 1.

\section{Positive feedback patterns}

Positive feedback means that tactile feedback is given to indicate that an interaction or task was performed correctly.

1) Robot motor is $\mathrm{ON}$
Action definition: robot motors are energized and the robot is ready to use but robot arm does not moves.

Feedback: Low-level vibration on all fingers for short time had been chosen after two times testing. It is inspired by chopped up road marking that alerts drivers by vibration on cars wheels. The operator is alerted that joint brakes are released and the robot is able to move.

TABLE I. VIBRATION PROPERTIES FOR EVENTS

\begin{tabular}{|c|c|c|c|c|}
\hline \multicolumn{2}{|c|}{ Robot action } & \multirow{2}{*}{$\begin{array}{l}\text { Vibration pattern } \\
\text { Low-level vibration on } \\
\text { all fingers }\end{array}$} & \multirow{2}{*}{$\begin{array}{c}\begin{array}{c}\text { Intensi } \\
\text { ty }\end{array} \\
25 \%\end{array}$} & \multirow{2}{*}{$\begin{array}{c}\begin{array}{c}\text { Quantity } \times \\
\text { duration }\end{array} \\
1 \times 0.5 \mathrm{~s}\end{array}$} \\
\hline \multirow{4}{*}{ 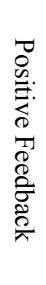 } & Motor ON & & & \\
\hline & Movement & $\begin{array}{l}\text { Vibration with same } \\
\text { intensity from little } \\
\text { finger to thumb }\end{array}$ & $25 \%$ & $5 \times 1 \mathrm{~s}$ \\
\hline & EndStop & Vibration on thumb & $60 \%$ & $1 \times 1.5 \mathrm{~s}$ \\
\hline & $\begin{array}{c}\text { Gripper } \\
\text { Close/Open }\end{array}$ & $\begin{array}{l}\text { Vibration on thumb and } \\
\text { index finger }\end{array}$ & $50 \%$ & $1 \times 1.2 \mathrm{~s}$ \\
\hline \multirow{4}{*}{ 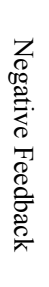 } & Collision & $\begin{array}{l}\text { High-level vibration on } \\
\text { all fingers }\end{array}$ & $70 \%$ & $1 \times 2.5 \mathrm{~s}$ \\
\hline & $\begin{array}{c}\text { Motor } \\
\text { overloaded }\end{array}$ & $\begin{array}{l}\text { Vibration fading (from } \\
\text { high-level vibration to } \\
\text { zero) }\end{array}$ & $\begin{array}{c}90 \%- \\
0 \%\end{array}$ & $1 \times 0.7 \mathrm{~s}$ \\
\hline & $\begin{array}{c}\text { Emergency } \\
\text { Stop }\end{array}$ & $\begin{array}{l}\text { High-level pulse on all } \\
\text { fingers }\end{array}$ & $70 \%$ & $5 \times 0.2 \mathrm{~s}$ \\
\hline & Singularity & Pulse on index finger & $40 \%$ & $5 \times 0.3 \mathrm{~s}$ \\
\hline
\end{tabular}

\section{2) Robot is moving toward EndStop}

Action definition: robot is executing a task and it's moving towards EndStop which is the final pose for the current task.

Feedback: The pattern of vibration is wave of vibration from little finger to the thumb. Intensity amplitude is changing according to the distance to the EndStop. This stimuli presents a feeling of movement and reaching to the destination in a natural and predictable way.

\section{3) Robot is in Endstop}

Action definition: when robot is in EndStop point, it means the task has been done successfully.

Feedback: vibration on thumb that is similar to well-known thumb up feedback has been selected as vibration patter. Vibration intensity and duration had been tuned with six attempts. This gives a natural "well done" impression for the operator.

\section{4) Gripper Close/Open}

Action definition: a gripper is attached to the robot arm to grab an object, which can be in two positions: opened or closed.

Feedback: Since people typically people their index finger and thumb to grab, vibration on index finger and thumb can provide similar sensation.

\section{Negative feedback patterns}


Negative feedback means that tactile feedback is given to indicate an error or mistake, requiring the user to repeat or correct the action.

\section{5) Collision happened}

Action definition: during the robot movement collision can happen between the robot arm and a physical obstacle in the environment.

Feedback: Non-stop, high-level vibration in all fingers can give the concept of this situation. The stimuli can be refined to give collision alert if collision avoidance device is installed on the robot.

\section{6) Robot motor is overloaded}

Action definition: when the robot motor is overloaded, motor will begin to slow down and draw more current than normal.

Feedback: ordinarily, when a motor of an electrical device is overloaded, it will work with fast speed for some seconds and then suddenly it will stop. Accordingly when robot motor overloaded, operator feels vibration fading in all fingers.

\section{7) Emergency stop pressed}

Action definition: emergency stop is a safety feature, it can be used when the robot has a potential to hurt humans or cause a hazardous accident.

Feedback: high-level pulse on all fingers can remind emergency situations. It is similar to fire alarm since gives a feeling of an unusual occurrence.

\section{8) Singularity}

Action definition: singularity is caused by the collinear alignment of two or more robot axes resulting in unpredictable robot motion and velocities.

Feedback: when robot is in singularity area, operator feels continues pulse on the index finger, since in everyday life the index finger is raised as a gesture of wrath or threatens. Entering the robot into the singularity area is result of the operator mistake hence pulse on his/her index finger reminds operator that he/she is in wrong track.

The system functionality of vibrotactile feedback solution was experimentally tested. The achieved results have been analyzed based on four variables: vibration intensity, duration, pattern and quantity. The vibration intensity is measured based on available power level for each vibration motor. The results showed an excellent performance for intensity (power level) parameter during all eight actions. Time was the most challenging variable in this system. General consideration of all results showed that time variable caused two difficulties in similar situations:

1) For five actions (motor ON, gripper open/close, collision, motor overloaded and emergency stop) stimulator utilized in a way that at least two vibration motors were desired to start working at the same time. Time variable had same behaviour for these five actions, vibration motors started working one by one, and the maximum delay between their starting times was 0.2 second.

2) The system had a weak point to produce more than one high-level pulse for short time period (0.2 - 0.3 second), this happened on two actions (singularity and emergency stop).

In general, the pattern variable performed successfully. Although in consequence of time variable performance, pattern variable did not follow the desired rhythm during some actions such as emergency stop.

\section{Virtual environment for remote operation}

The system consists of a local room and a remote room. The local was represented by a virtual room where the industrial robot model is available. The remote operator who wears the vibrotactile glove is situated in the remote room.

The virtual room is created in VirCA environment. VirCA is a loosely coupled modular, 3D Internet based interactive virtual environment for collaborative manipulation of robots and other hardware or software equipment [13]. The interaction with the users provides various forms of communication channels from 3D menus to speech recognition and text to speech engine $[14,15]$. This system is open for new devices; thus for the integration of the vibrotactile glove in a robotic system.

The virtual room included a KUKA 30 industrial robot arm and nine posters on the wall with different texts and images [16]. The physical KUKA 30 industrial robot arm is located at NUC's laboratory. The actions and events for tactile feedback were part of the virtual robot device the vibrotactile glove was connected to these signals. This way the user with the glove on the hand was an outsider to the virtual reality: had limited vision and partial information of the system. The vibrotactile feedback was used to support the remote operator with additional information, and the intuitiveness of this feedback was tested.

\section{USABILITY TEST FOR VIBROTACTILE FEEDBACK}

A total of 12 participants were from UiT - The Arctic University of Norway. From these twelve participants 7 are male and 5 are female with ages ranging from 20 to 32 resulting in an average of 26 years old. All participants have a good understanding of the English language.

9 over the 12 participants were well versed in the field of robotics, while the rest were not familiar to it. Thus, the test could be considered a mixture of non-expert and expert review. An expert reviewer could already have a good idea of how to accomplish the task. The reason for the use of random people who are not familiar to the subject is that one of the usability testing goals is to evaluate the intuitiveness of the vibrotactile glove system for both experts and non-experts.

The participants' gender, age education level and background is summarized in Table 2. All of the participants have met some kind of computer simulator previously. 4 participants are active players in First Person Shooter (FPS) type games and console games. 
The testing was carried out at the Visualization Center at UiT - The Arctic University of Norway laboratory. This room is equipped with a screen of 6.5 meters by 1.5 meters (width $\times$ height). A mouse was available for the user to interact with the controller device and to perform robot actions. The vibrotactile glove system was available for the participants to sense the vibrotactile feedback. The general view for the participants, when they entered the room can be seen in Figure 3.

TABLE II. PARTICIPANT DATA

\begin{tabular}{|l|llll|}
\hline & Gender & Age & Education level & Education background \\
\hline Participant 1 & Male & 29 & Master & Design Engineering \\
Participant 2 & Male & 24 & Master & Industrial Engineering \\
Participant 3 & Male & 27 & Master & Industrial Engineering \\
Participant 4 & Female & 28 & Bachelor & Civil Engineering \\
Participant 5 & Male & 25 & Master & Industrial Engineering \\
Participant 6 & Male & 25 & Master & Industrial Engineering \\
Participant 7 & Female & 30 & Master & Electrical Engineering \\
Participant 8 & Male & 32 & Bachelor & Civil Engineering \\
Participant 9 & Female & 20 & Bachelor & Electrical Engineering \\
Participant 10 & Female & 26 & Master & Industrial Engineering \\
Participant 11 & Female & 28 & Master & Industrial Engineering \\
Participant 12 & Male & 30 & Bachelor & Industrial Engineering \\
\hline
\end{tabular}

The test conductor as a local operator executed eight available robot actions and participant as remote operator sensed vibrotactile feedback. All the participants have executed the test one by one. They were not allowed to discuss experiences, while waiting in a separate room. When a participant entered the laboratory, he/she was greeted by the conductor and the goal of the test, the procedure and the capabilities of the system were described.

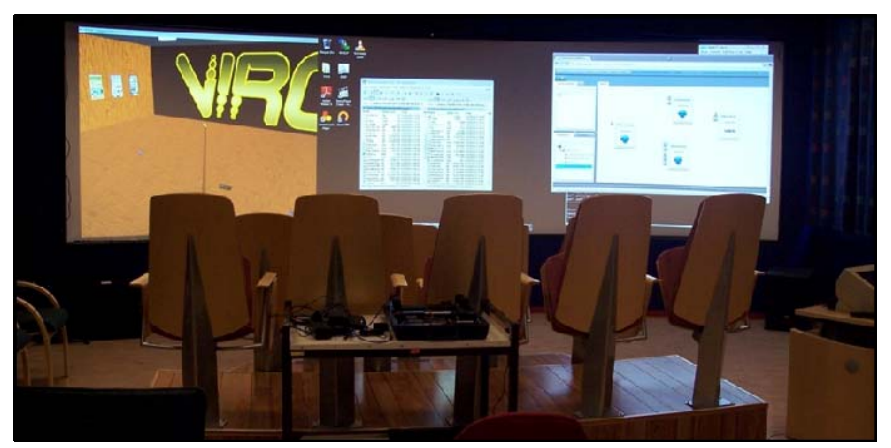

Fig. 3. Overview of the Visualization Center at UiT - The Arctic University of Norway

In order to interpret different types of vibrotactile stimuli, the participants needed to be trained. Since they were selected to be intuitive, the training time was short. The participant was informed that he/she can abort at any time and after the test he /she should fill out a questioner and will have the possibility to discuss his/her feeling about the experiment.

When all tasks were completed, the participants were given questionnaires to answer and in the end there was a free discussion for participants in order to express their recommendation for further improvement.
A questionnaire was constructed in such a manner to avoid responses that do not require thinking. The main issues examined during the usability tests were:

- How the participant responded when the vibrotactile glove system started working?

- How participant rates the intuitiveness and logic behind each vibrotactile stimuli?

- Measurement of simplicity in distinction of the different vibrotactile stimuli for the participants.

- Evaluating necessary time for the participants to reach a level of proficiency in using the system without being confused.

- Clarifying the general impressions of the system for the participants.

Rating scale in the questionnaire form was defined from 1 (Poor) to 5 (Excellent).

\section{A. Results}

Due to many similar observations of the participants during the usability tests, some of the results will be presented in general terms.

The average rate for the logic behind the vibrotactile stimuli was 4.3 . The participants expressed that they were able to memorize the stimulator feedback at first attempt. It proved that vibrotactile stimulator had been utilized as an intuitive feedback solution for the robot actions.

Two of the participants reported difficulties to distinguish between the Motor ON and Collision feedback pattern.

Regarding the participant's proficiency level in using the system, it was observed that the 9 participants who are well versed in the field of robotics were able to complete the task in first attempt while the 3 non-expert participants increased their efficiency after two attempts and reach the required proficiency level in third attempt. It is confirmed that the application of vibrotactile feedback is an intuitive feedback solution.

TABLE III. VIBROTACTILE FEEDBACK USABILITY TESTING RESULT

\begin{tabular}{|lcccc|}
\hline \multicolumn{1}{|c}{ Robot action } & $\begin{array}{c}\text { Stimulator } \\
\text { logic }\end{array}$ & $\begin{array}{c}\text { Simplicity } \\
\text { to } \\
\text { distinguish }\end{array}$ & $\begin{array}{c}\text { System } \\
\text { intuitiveness }\end{array}$ & $\begin{array}{c}\text { General } \\
\text { impression }\end{array}$ \\
\hline Motor ON & 4 & 3 & 4 & 5 \\
Movement & 5 & 5 & 5 & 5 \\
Gripper & 5 & 5 & 4 & 5 \\
Close/Open & 5 & 5 & 5 & 4 \\
EndStop & 3 & 3 & 3 & 4 \\
Collision & 5 & 5 & 4 & 5 \\
Motor Overloaded & 2 & 5 & 3 & 3 \\
Singularity & 5 & 5 & 5 & 5 \\
Emergency stop & $\mathbf{4 . 3}$ & $\mathbf{4 . 5}$ & $\mathbf{4 . 1}$ & $\mathbf{4 . 5}$ \\
\hline Average rate & & &
\end{tabular}

The three non-expert participants reported confusion during the testing period for singularity action, this may be because 
these participants had problem to understand the concept of singularity in robotics.

In general terms, all participants described their impression of the system as to be easy to use and the average of rate was 4.5. This means that from the usability point of view the vibrotactile feedback solution is in the acceptability range, however further improvements are recommended.

Table 3 presents the summarized results from the individual assessments of the tests.

\section{B. Discussion}

The vibrotactile feedback solution had been successfully performed for all the participants in the usability tests. Although observations exposed the system would be more intuitive and efficient if it was combined with multimedia system. There are arguably many approaches for addressing this issue. One possible approach is a combination of the available system with sound. The challenges associated with this approach could be this system will be effective only for a quiet working environment. Other approaches could be having pop-up text messages or light effects on the screen. This approach would not have the limitation of working environment type.

- Following propositions are made from the observations during tests and free discussion session with the participants:

- The vibrotactile feedback solution was intuitive to understand.

- The system would be more intuitive and efficient if it was combined with multimedia system such as pop-up text messages on the screen, light or sound.

- Participant's proficiency level in using the system can be achieved with minimal training.

- Distinguish the difference between stimulators can be confusing for non-expert operators.

- The vibrotactile glove system in the industrial implementation recommended to being available for both left handed and right handed operators.

The result from the experimental confirmed that application of vibrotactile feedback in remote operation is an adequate solution. It provides successful and efficient communication with a remote operator in order to support to industrial robot systems. Moreover, it shows that the use of the system can be successfully applied to any multivariable system that requires human supervisory.

\section{CONCLUSIONS}

A vibrotactile device and vibration patterns for industrial robot remote operation feedback was presented. The patterns are constructed based upon cognitive and intuitive disciplines.
Usability tests with experienced and inexperienced users were conducted. Results show that in fact the patterns are capable of transferring robot cell variables intuitively for remote users. Extending the communication channel to multimodality is considered in the future.

\section{ACKNOWLEDGMENT}

The authors would like to thank Azin Aryania for the development and executing the usability tests.

\section{REFERENCES}

[1] Michael A. Goodrich and Alan C. Schultz: Human-robot interaction: a survey, Found. Trends Hum.-Comput. Interact., Volume 1, Issue 5, January 2007, pp. 203-275.

[2] Brian Vaughan, Jing Guang Han, Emer Gilmartin, Nick Campbell: Designing and Implementing a Platform for Collecting Multi-Modal Data of Human-Robot Interaction, Acta Polytechnica Hungarica, Volume 9, Issue 1, 2012, pp.7-17.

[3] World Robotics - Industrial Robots 2012, Executive Summary. (http://www.worldrobotics.org)

[4] Fekete Róbert Tamás, Fazakas Tamás, "3D Reconstruction System for Autonomous Robot Navigation", In: 11th IEEE International symposium on Computer Intelligence and Informatics, 2010, pp. 267-270.

[5] T. Thomessen, T. Kosicki: Cognitive audio-visual infocommunication applied in remote support for industrial robot systems. Cognitive Infocommunications (CogInfoCom), 2nd International Conference on, 7-9 July 2011, pp.1-5.

[6] Fredrik Reme: Advanced Remote Control of Industrial Robots, Master Thesis, Norwegian University of Science and Technology (NTNU), Department of Production and Quality Engineering in cooperation with PPM AS, 2012.

[7] Grigore Burdea, Grigore C. Burdea: Force and touch feedback for virtual reality. New York, Wiley, 1996.

[8] R.A.Russel: Robot Tactile Sensing. Prentice Hall, New York, 1990.

[9] Péter Galambos: Vibrotactile Feedback for Haptics and Telemanipulation: Survey, Concept and Experiment, Acta Polytechnica Hungarica, Vol. 9, No. 1, 2012, pp. 41-65.

[10] G. Sziebig, B. Solvang, C. Kiss, P. Korondi: Vibro-tactile feedback for VR systems, Human System Interactions, 2009, pp. 406-410.

[11] Bethopedia, online (http://wiki.bethanycrane.com/somaticsenses)

[12] Y. Muramatsu, M. Niitsuma, T. Thomessen: Perception of Tactile Sensation Using Vibrotactile Glove Interface. 3rd IEEE International Conference on Cognitive Infocommunications, 2012, pp. 621-626.

[13] Galambos Péter, Baranyi Péter: VirCA as Virtual Intelligent Space for RT-Middleware, in Proceedings of 2011 IEEE/ASME International Conference on Advanced Intelligent Mechatronics (AIM2011), 2011, pp. 140-145.

[14] Dávid Vincze, Szilveszter Kovács, Márta Gácsi, Péter Korondi, Ádám Miklósi, Péter Baranyi: A Novel Application of the 3D VirCA Environment: Modeling a Standard Ethological Test of Dog-Human Interactions, Acta Polytechnica Hungarica, Vol. 9, No. 1, 2012, pp. 107 120.

[15] Péter Baranyi, Géza Németh, Péter Korondi: 3D Internet for Cognitive Infocommunication, INFORMATIKA - A GÁBOR DÉNES FÖISKOLA KÖZLEMÉNYEI, Vol. 12, No. 2, 2010, pp. 3-6.

[16] G. Sziebig, T. Øritsland: Navigating in 3D Immersive Environments: a VirCa usability study. 10th IFAC Symposium on Robot Control International Federation of Automatic Control Vol. (10), 2012, pp. 380384. 